International Journal of Computer Science \& Information Technology (IJCSIT) Vol 4, No 2, April 2012

\title{
A Semi-Blind Reference Watermarking Scheme Using DWT-DCT-SVD for Copyright Protection
}

\author{
Satyanarayana Murty. $\mathrm{P}^{1}$, M.Uday Bhaskar ${ }^{2}$, P. Rajesh Kumar ${ }^{3}$ \\ ${ }^{1}$ Sr.Associate Professor, Department of ECE, GIITS, Vishakapatnam, India \\ murty_pattisapu@yahoo.co.in \\ ${ }^{2}$ PG Student, Department of CSE,Sri Sai Aditya Institute Of Science \& Technology, \\ Andhra Pradesh, India \\ mangipudiuday@gmail.com \\ ${ }^{3}$ Associate Professor, Department of ECE, AU college of Engineering, Vishakapatnam, \\ India
}

\begin{abstract}
In this paper we propose a semi-blind watermarking scheme using Discrete Wavelet Transform, Discrete Cosine Transform and Singular Value Decomposition for copyright protection. We used a gray scale image as a watermark to hide in another gray scale image as a cover image. The cover image is modified (Zig-Zag) and divided to number of blocks of size $n \times n$. We find the spatial frequency of each block and kept a threshold on this spatial frequency to form a reference image. Then the reference image is transformed into wavelet domain. A DCT is applied to the HF band of DWT decomposition reference image. We hide the watermark into reference image by modifying the singular values of transformed DCT coefficients with the singular values of watermark. The proposed algorithm provides a good imperceptibility and robust for various attacks.
\end{abstract}

KEYWORDS

Spatial frequency, DWT, DCT, SVD, Zig_Zag, Reference image.

\section{INTRODUCTION}

Due to the rapid and extensive growth of network technology, digital information can now be distributed much faster and easier. However, according to the insufficient cognizance of intellectual property, the condition of illegal copies and spread of copyright reserved information are growing serious. To protect the copyright of multimedia information and to decrease the impulse to copy and spread copy right reserved multimedia information. Fortunately, the digital watermarking technique is the right choice to protect the right full information of the owners. Watermarking has two processes. One is embedding; a mark or signal is hiding into multimedia content as cover information. Second one is extraction; the hidden mark or signal at receiving end.

In digital image watermarking the inserted watermark should not degrade the visual perception of an original image. The first method for hiding watermarking is by directly changing original cover-media. The advantages are simple and fast calculated but cannot protect itself from varied signal processing attacking $[1,2,3]$. The most of watermarking techniques embed the information data in the coefficients of transformation domain of the cover image, such as Fourier transformation, discrete cosine transformation, wavelet transformation and singular value decomposition. Image watermarking algorithms using Discrete Cosine Transform (DCT) [4, 5, 6, 12, 13], Discrete Wavelet Transform (DWT) $[7,8,9,10,11]$ Singular Value Decomposition (SVD) $[14,15]$ are available in the literature.

DOI : $10.5121 /$ ijcsit.2012.4206 
Domain transformation watermarking schemes, in general, first use DCT and DWT and then transforms the image into the spatial domain. Watermarking schemes usually uses a watermarking on black and white or greyscale images. Hybrid domain transforms are also available in the literature DCT- SVD [16,17,18] and DWT-SVD [19,20,21,22,23,24,25,26,27]. In this paper we proposed an optimal watermarking technique based on DWT- DCT-SVD. The rest of the paper is organized as follows: Section 2 describes the related work, while Section 3 contains our proposed algorithm and in section 4 experimental results followed by conclusions in Section 5.

\section{The DCT transform:}

The Discrete Cosine Transform (DCT) is a technique that converts a spatial domain waveform into its constituent frequency components as represented by a set of coefficients. These transforms are the members of real-valued discrete sinusoidal unitary transforms. The DCT has excellent energy compaction for highly correlated data. A 2D DCT can be computed as two separate one-dimensional transforms. This is shown in equation 1. The DCT transformed coefficients have a Dc component and AC component. The AC components are used for watermarking embedding, which provides good robust against various attacks.

$$
C(u, v)=\alpha(u) \alpha(v) \sum_{x=0}^{N-1} \sum_{y=0}^{N-1} f(x, y) \cos \left[\frac{(2 x+1) u \pi}{2 N}\right] \cos \left[\frac{(2 y+1) v \pi}{2 N}\right]
$$

$\mathrm{f}$ or $\mathrm{u}, \mathrm{v}=0,1,2, \ldots \mathrm{N}-1$.

Where

$$
\begin{aligned}
& \alpha(u)=\left\{\begin{array}{cl}
\frac{1}{\sqrt{2}} & \mathrm{u}=0 \\
1 & \mathrm{u}=1,2, \ldots \ldots . \mathrm{N}-1
\end{array}\right. \\
& \alpha(v)=\left\{\begin{array}{cl}
\frac{1}{\sqrt{2}} & \mathrm{v}=0 \\
1 & \mathrm{v}=1,2, \ldots \ldots . \mathrm{N}-1
\end{array}\right.
\end{aligned}
$$

\section{The DWT transform:}

The Discrete Wavelet Transform (DWT) is obtained by filtering the signal through a series of digital filters at different scales. The scaling operation is done by changing the resolution of the signal by the process of sub sampling. The analysis filter bank consists of a low-pass and highpass filter at each decomposition stage. When the signal passes through these filters, it splits into two bands. The low-pass filter, which corresponds to an averaging operation, extracts the coarse information of the signal. The high-pass filter, which corresponds to a differencing operation, extracts the detail information of the signal. The output of the filtering operation is decimated by two. A two dimensional transform is accomplished by performing two separate one-dimensional transforms. First, the image is filtered along the row and decimated by two. It 
International Journal of Computer Science \& Information Technology (IJCSIT) Vol 4, No 2, April 2012

is then followed by filtering the sub-image along the column and decimated by two. This operation splits the image into four bands, namely, LL, LH, HL and HH respectively as shown figure 1 .

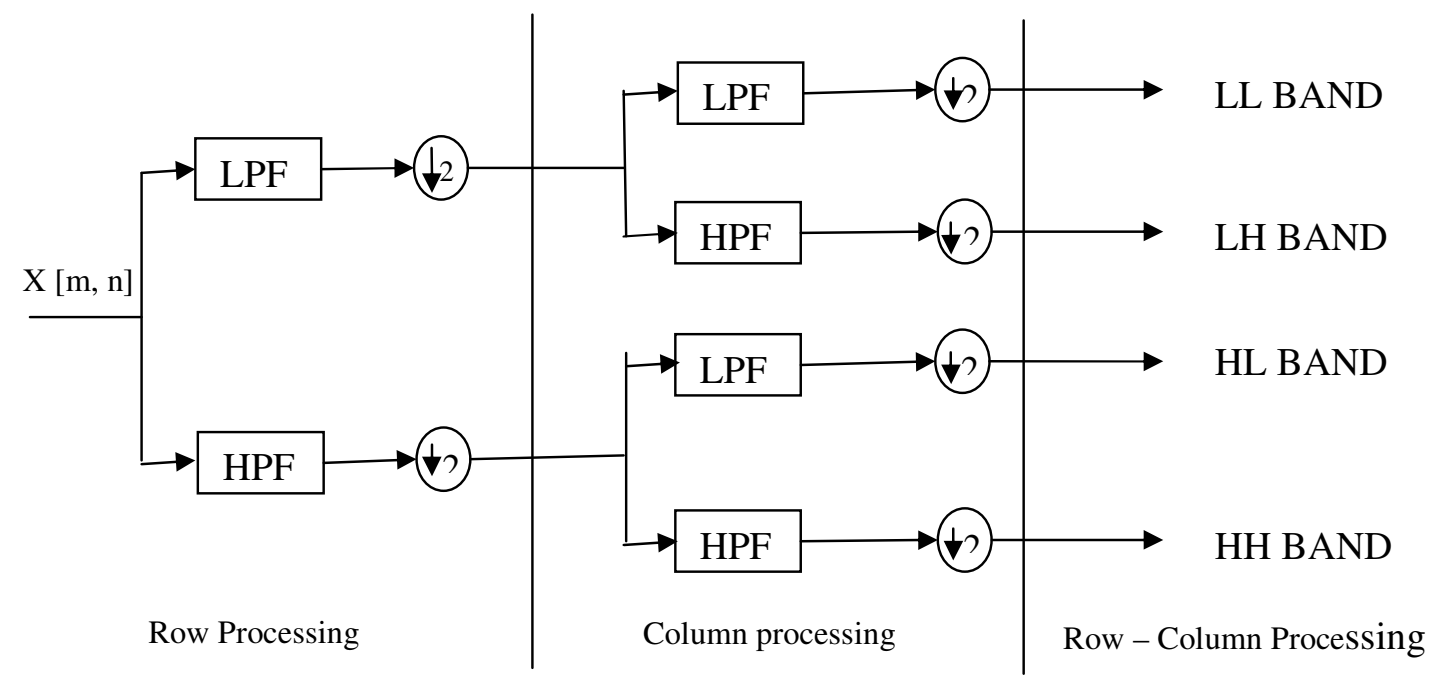

\section{Singular Value Decomposition:}

Figure - 1

In linear algebra, the singular value decomposition (SVD) is an important factorization of a rectangular real or complex matrix, with several applications in signal processing and statistics. The SVD of rectangle matrix $\mathrm{A}$ is a decomposition of the form

$$
A=U S V^{T}
$$

Where $\mathrm{A}$ is an $\mathrm{m} \times \mathrm{n}$ matrix. $\mathrm{U}, \mathrm{V}$ are orthogonal matrices. $\mathrm{S}$ is a diagonal matrix composed of singular values of $A$. The singular values $S_{1} \geq S_{2} \geq S_{3} \ldots \ldots \geq S_{n} \geq 0$ appear in descending order along the main diagonal of $\mathrm{S}$.

\section{Spatial Frequency}

Spatial frequency of an image can be used to know the overall activity level in an image [9]. For an image block $l_{1}$ of size $\mathrm{M} \times \mathrm{N}$, the spatial frequency is defined as:

$$
S F=\sqrt{R F^{2}+C F^{2}}
$$

Where RF and CF are the row and column frequencies and are defined as:

$$
\begin{aligned}
& R F=\sqrt{\frac{1}{M_{1} N_{1}} \sum_{m=1}^{M 1} \sum_{N=1}^{N 1}[l(m, n)-l(m, n-1)]^{2}} \\
& C F=\sqrt{\frac{1}{M_{1} N_{1}} \sum_{m=1}^{M 1} \sum_{N=1}^{N 1}[l(m, n)-l(m-1, n)]^{2}}
\end{aligned}
$$


International Journal of Computer Science \& Information Technology (IJCSIT) Vol 4, No 2, April 2012

\section{RELATED WORK}

S S Bedi proposed a new SVD based and DCT-DWT oriented digital watermarking scheme. The middle band DCT coefficients are chosen to achieve high robustness against JPEG compression. He used a LL band of DWT to insert a watermark.[ 28 ]. Navas K A proposed a DWT-DCT-SVD watermarking algorithm, which over comes the problems of traditional watermarking. [ 29 ]. In this paper, they proposed a robust watermarking technique which combines features of discrete wavelet transformation (DWT), discrete cosine transformation and singular value decomposition. In this technique DWT is used to decompose the colour images into various frequency and time scale. Block DCT is applied on DWT coefficients of various frequencies to provide high level of robustness. DCT transformed blocks of size $4 \times 4$ are further decomposed using dual SVD technique to get singular values in which watermark is to be hidden.[ 30 ].

In this paper the authors proposed an algorithm which combines the advantages DWTDCT-SVD. Also they used the Arnold transform before embedding the watermark into original image. This improves the robustness of algorithm. [31]. A novel watermarking algorithm for digital image based on DWT-DCT-SVD was proposed in this paper. They applied four layers DWT on the original image and choose the low-frequency sub image and three high frequency sub images of the fourth layer. Then, by using DCT, SVD and the decomposition criteria proposed in this paper, they embedded the low-frequency sub-image and the three high frequency sub images obtained watermarking image into those of the original image adaptively.[ 32]. In this paper, authors presented a hybrid watermarking scheme based on DWT-DCT-SVD. They used the individual advantages of DWT, DCT and SVD. So they got good imperceptibility and robustness against various attacks. [33].

\begin{tabular}{|c|c|c|c|c|c|c|c|c|}
\hline Images & lake & Lena & Bridge & Lvroom & Boat & Mandrill & Pirate & Peppers \\
\hline PSNR & 48.35 & 47.42 & 48.40 & 48.98 & 44.41 & 44.97 & 45.06 & 44.20 \\
\hline \multicolumn{10}{|c|}{ Table - 3 PSNR values } \\
\hline
\end{tabular}

\section{PROPOSEDALGORITHM}

\subsection{Watermark Embedding}

The Watermark Embedding Procedure as Shown in Figure 2(a) 
International Journal of Computer Science \& Information Technology (IJCSIT) Vol 4, No 2, April 2012

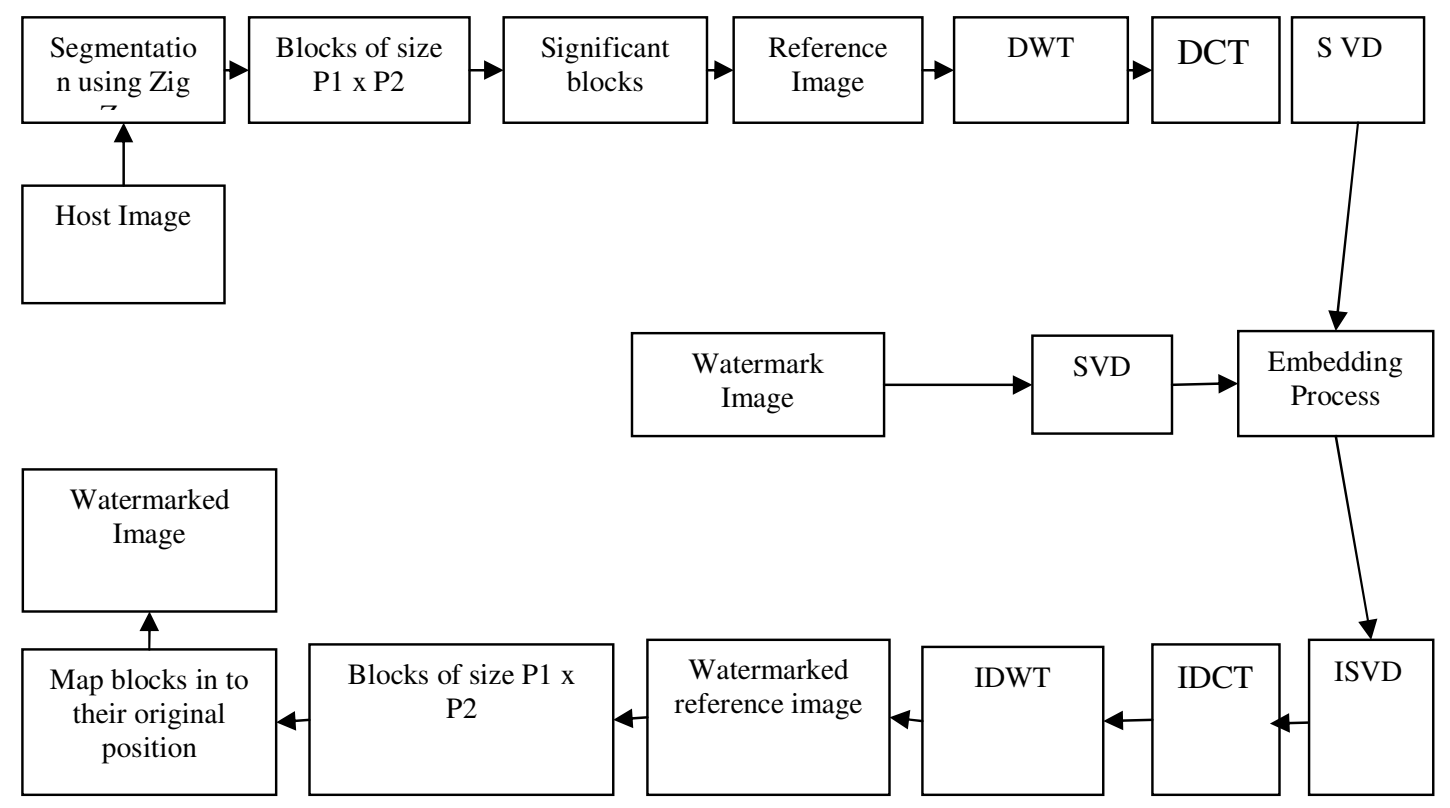

Figure 2(a)

First, the original image is segmented into blocks of size $\mathrm{p}_{1} \times \mathrm{p}_{2}$ via ZIG_ZAG sequence denoted by $\mathrm{F}^{\mathrm{l}}$, where 1 is the number of blocks.

Step1: Find out the spatial frequency in each block, denoted by $\mathrm{SF}_{\mathrm{F}} \mathrm{l}$.

Step2: Spatial frequencies of each block are stored in descending order. Then make a threshold on spatial frequency. Those blocks, which have spatial frequency less than or equal to threshold, are considered as significant blocks and are used for making reference image, $\mathrm{F}_{\text {ref }}$ which is a size of $m \times n$.

Step3: Perform DWT on the reference image, which is denoted by $f_{d w t}$.

Step4: Perform DCT on the $\mathrm{f}_{\mathrm{dwt}}$ HL band of DWT decomposition, which is denoted by $\mathrm{f}_{\mathrm{dct}}$

Step4: Perform SVD transform as shown in equation (5).

$$
f_{\text {svd }}^{r e f}=U_{\text {fref }} * S_{\text {fref }} * V_{\text {fref }}^{T}
$$

Step5: Perform SVD on watermark image as shown in equation (6).

$$
W_{s v d}=U_{w} * S_{w} * V_{w}^{T}
$$

Step6: Modify the single values of reference image with the singular values of watermark as

$$
\left(\sigma_{\text {fref }}\right)^{*}=\sigma_{\text {fref }}+\beta * \sigma_{w}
$$

where $\beta$ gives the watermark depth.

Step7: Perform inverse SVD,

$$
f_{\text {isvd }}^{*}=U_{\text {fref }} * S_{\text {fref }}^{*} * V_{\text {fref }}^{T}
$$

Step8: Perform inverse DCT and DWT to construct the modified reference image, denoted by $f_{r e f}^{*}$. Again $f_{r=f}^{*}$ is segmented into blocks of size $\mathrm{p}_{1} \times \mathrm{p}_{2}$ and mapped onto their original positions for constructing the watermarked image. 
International Journal of Computer Science \& Information Technology (IJCSIT) Vol 4, No 2, April 2012

We save the positions of the significant blocks and reference image for the extraction process.

\subsection{Watermark Extraction}

The Watermark Extraction Procedure as Shown in Figure 2(b)

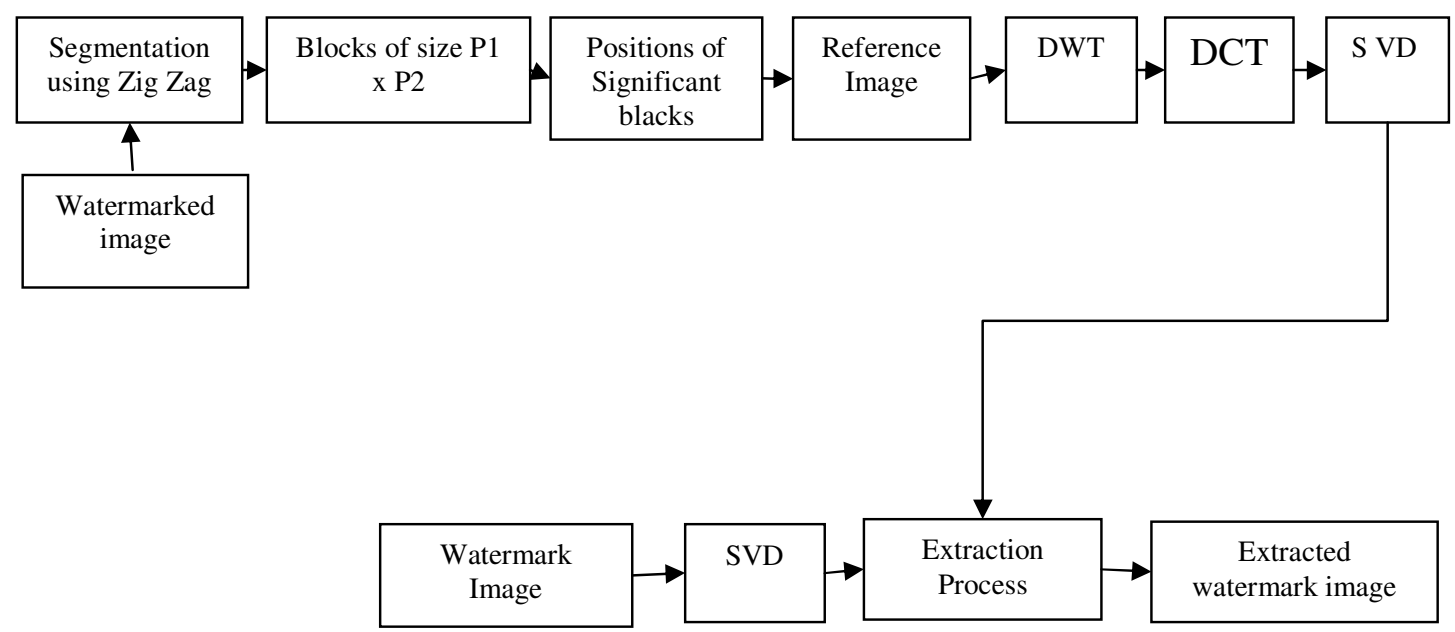

Figure 2(b)

The objective of the watermark extraction is to obtain the estimate of the watermark. For watermark extraction, original reference and watermarked images, left and right singular vectors must be available at the receiver end.

Step1:Using the positions of significant blocks, make the reference image from the watermarked image, denoted by $F_{r \bullet f}^{W}$.

Step2: Perform DWT and DCT on watermarked reference, $F_{r e f}^{W}$. which is denoted by $f_{r e f}^{W}$.

Step3: Perform SVD transform on both $f_{r e f}$ and $f_{r e f}^{W}$.

$$
\begin{gathered}
f_{\text {ref }}=U_{\text {fref }} * S_{\text {fref }} * V_{\text {fref }}^{T} \\
f_{\text {ref }}^{W}=U_{\text {fref }}^{W} * S_{\text {fref }}^{W} * V_{\text {fref }}^{T_{W}}
\end{gathered}
$$

Step4: Extract the singular values of the watermark.

$$
\sigma_{W}^{e x t}=\frac{\sigma_{\text {fref }}^{W}-\sigma_{\text {fref }}}{\beta}
$$

Step5: Obtain the extracted watermark as:

$$
W^{e x t}=U_{W} * S_{W}^{e x t} * V_{w}^{T}
$$

\section{RESULT ANALYSIS}

\section{Imperceptibility Performance}

Imperceptibility means that the perceived quality of the image should not be distorted by the presence of the watermark. The peak signal to noise ratio (PSNR) is typically used to measure the degradation between original image and watermarked image. 
International Journal of Computer Science \& Information Technology (IJCSIT) Vol 4, No 2, April 2012

$$
\begin{gathered}
R M S E=\sqrt{\frac{[f(i, j)-F(i, j)]^{2}}{N^{2}}} \\
P S N R=20 \log \frac{255}{R M S E} d b
\end{gathered}
$$

RMSE Is the Root Mean Square Error and is a comparison between the host image and watermarked image.

$\mathrm{f}(\mathrm{i}, \mathrm{j})$ and $\mathrm{F}(\mathrm{i}, \mathrm{j})$ represent host and watermarked images respectively. Size of the host image is $\mathrm{N} \times \mathrm{N}$.

\section{Robustness Performance}

Robustness of a watermarking algorithm is that the embedded data should survive any signal processing operation the host signal goes through and preserve its fidelity. The similarity between the original watermark and the extracted watermark from the attacked watermarked image was measured by using the correlation factor $\rho$, which is computed using the following Equation:

$$
\rho(w, \widetilde{w})=\frac{\sum_{i=1}^{N} w_{i} \widetilde{w}_{i}}{\sqrt{\sum_{i=1}^{N} w_{i}^{2}} \sqrt{\sum_{i=1}^{N} \widetilde{w}_{i}^{2}}}
$$

Where $\mathrm{N}$ is the number of pixels in watermark, $\mathrm{w}$ and $\widetilde{w}$ is the original and extracted watermarks respectively. The correlation factor $\rho$, may take values between -1 and 1 .

The algorithms discussed in the above section have been implemented in MATLAB for the gray scale Boat, Lena, Bridge, Living Room, Lake, Mandrill, Pirate and Pepper images of size $512 \times 512$. For watermark, copyright gray scale image and cameramen gray scale image of size $128 \times 128$ was used. The original images Boat, Lena, Bridge and Living Room were watermarked with cameramen, while the other original images Lake, Mandrill, Pirate and Pepper were watermarked with copyright image. In our experiment, the size of blocks is taken to be $8 \times 8$. The original images were shown in table -1 . The watermarked images were shown in table -2 . In table 2 (a) shows Watermarked Images with watermark as cameramen image and (b) shows Watermarked Images with watermark as copyright image. The corresponding PSNR values are showed in table -3 . The original watermark and the extracted watermark (without applying attacks) images and normalized cross correlation values are shown in table 4 and table -5 respectively.

We investigate the robustness of the algorithm by considering Average filtering, Median filtering, Compression, Cropping, Gaussian noise, Histogram Equalization, Resize, Rotate, Pixilation, Sharpening, wrapping and motion blur attacks. After these attacks on the watermarked image, we have compared the extracted watermarks with the original one. 
International Journal of Computer Science \& Information Technology (IJCSIT) Vol 4, No 2, April 2012
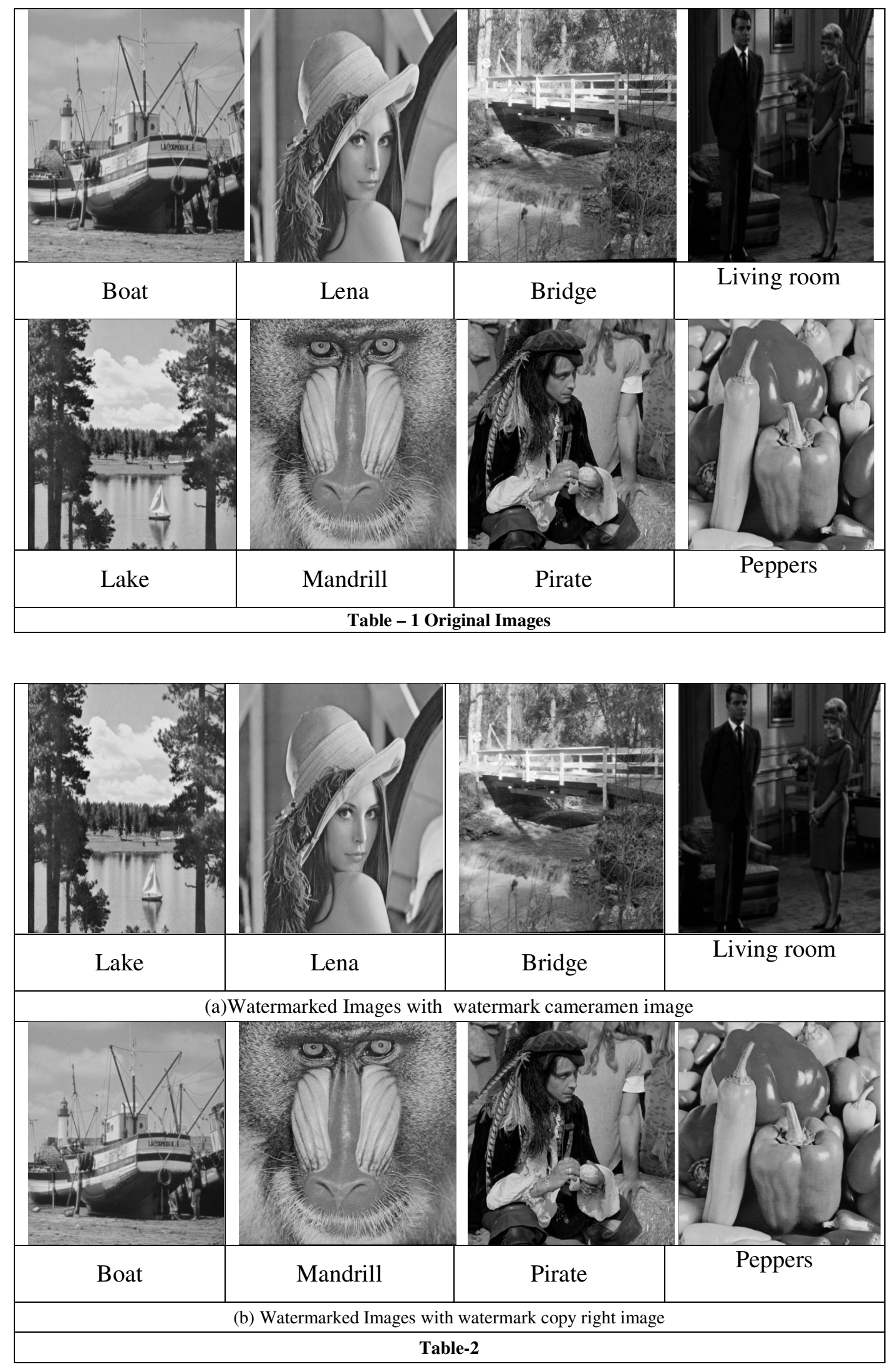


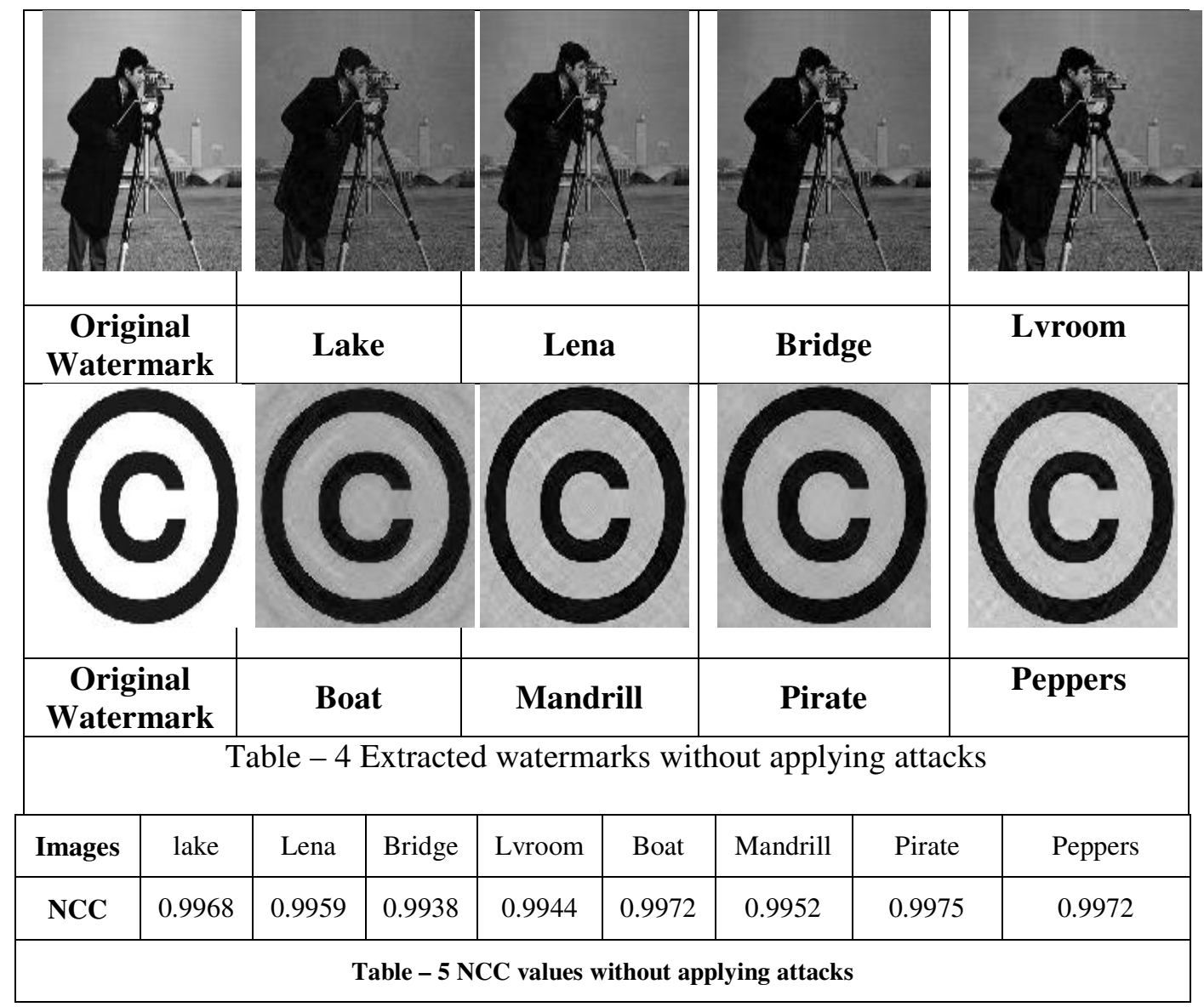

\section{Different types of attacks}

\section{Average Filtering and Median Filtering}

The most common manipulation in digital image is filtering. The extracted watermarks, after applying $13 \times 13$ average and median filtering, are shown in figure 3 . It can be observed that after applying these filters, images are very much degraded and lot of data is lost but the extracted logo watermark is still recognizable.

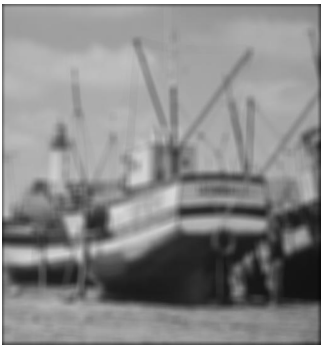

( a)

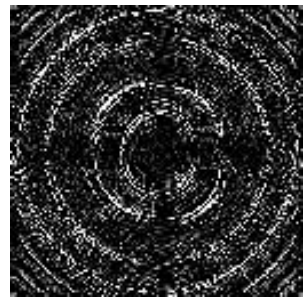

(b)

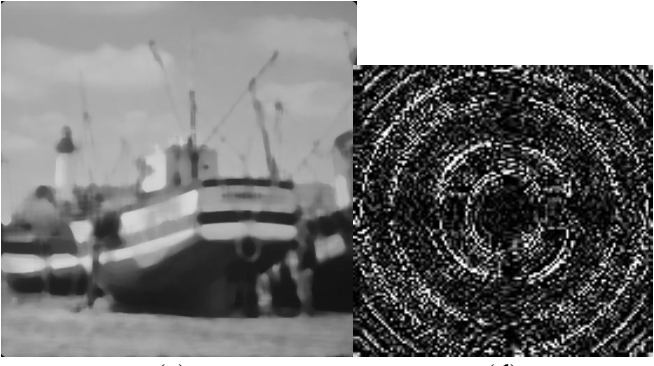

(c) (d)

Fig 3 (a) watermarked boat image after $13 \times 13$ average filtering (b) extracted watermark (c) watermarked boat image after $13 \times 13$ median filtering (d) extracted watermark 


\section{Gaussian filter (75\%) and Compression (80: 1)}

To verify the robustness of the watermarking scheme, another measure is noise addition. In real life, the degradation and distortion of the image come from noise addition. In our experiment, we have added $75 \%$ additive Gaussian noise in the watermarked image. The attacked watermarked image and the extracted watermark are shown in figure-4 (a) and (b) respectively. In real life applications, storage and transmission of digital data, a lossy coding operation is often performed on the data to reduce the memory and increase efficiency. Hence we have also tested our algorithms for the JPEG compression (80:1). The attacked watermarked image and the extracted watermark are shown in figure-4 (c) and (d) respectively.

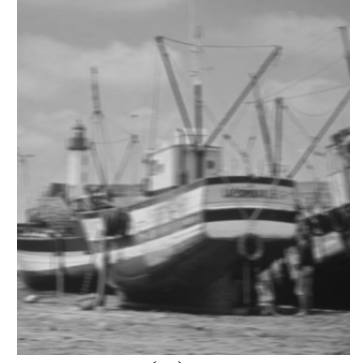

(a)

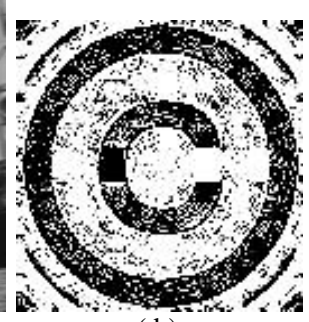

(b)

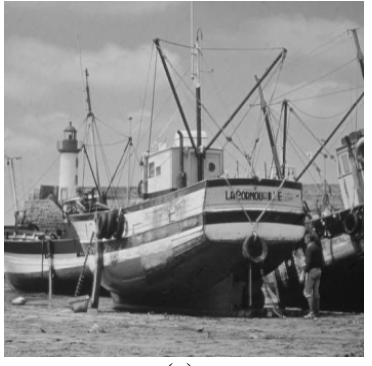

(c)

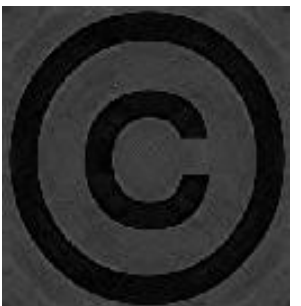

(d)

Fig 4 (a) watermarked boat image after adding 75\% additive Gaussian noise (b) extracted watermark

(c) watermarked boat image after jpeg compression image(80:1) (d) extracted watermark

\section{Cropping (25\% reaming) and Rotation $\left(\mathbf{5 0}^{\circ}\right)$}

Image cropping is very frequently used in real life. Cropping an image is done by either hiding or deleting rows or columns. This is a lossy operation. For this attack, $75 \%$ of the watermarked image is cropped and then watermark is extracted. The attacked watermarked image and the extracted watermark are shown in figure-5 (a) and (b) respectively. We have also tested our algorithms for rotation. The attacked watermarked image and the extracted watermark are shown in figure-5 (c) and (d) respectively.

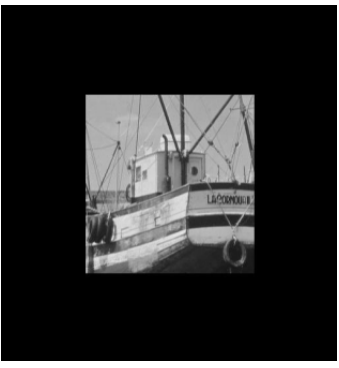

( a)

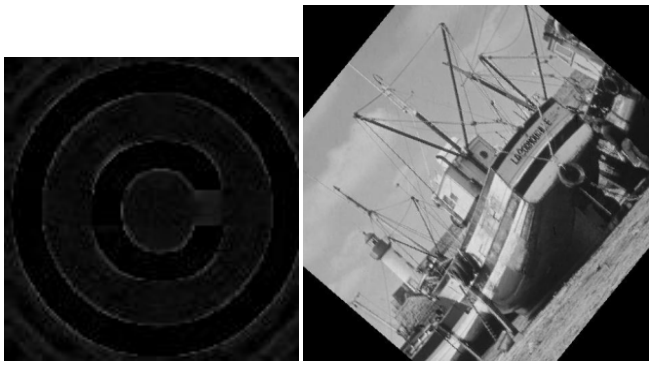

( b) (c)

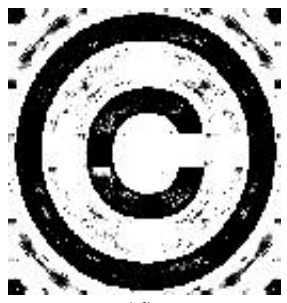

(d)

Fig 5 (a) watermarked boat image after cropping (b) extracted watermark (c) watermarked boat image after rotation (d) extracted watermark

\section{Histogram and Pixilated (3)}

The watermarked image is exposed for histogram equalization attack. The attacked watermarked image and the extracted watermark are shown in figure-6 (a) and (b) respectively. Pixilation (mosaic) is another disturbing operation on watermarked image to eliminate or destroying the watermark. The corresponding attacked watermarked image and the extracted watermark are shown in figure-6 (c) and (d) respectively. 
International Journal of Computer Science \& Information Technology (IJCSIT) Vol 4, No 2, April 2012

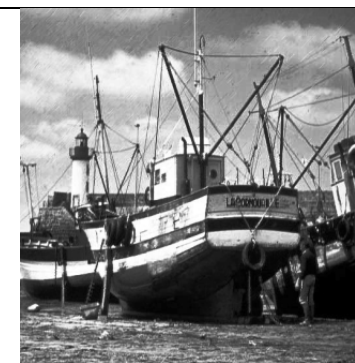

( a)

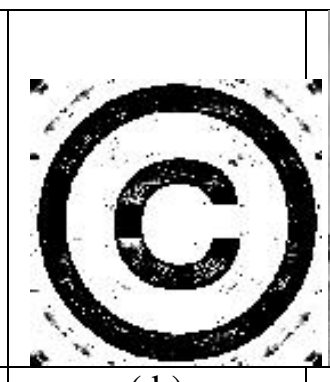

(b)

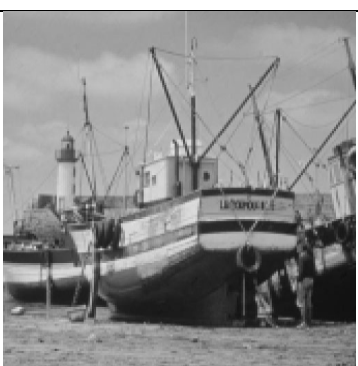

(c)

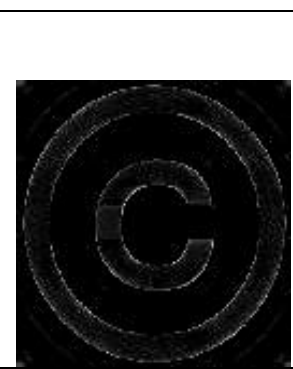

(d)

Fig 6 (a) watermarked boat image after histogram equalization (b) extracted watermark (c) watermarked boat image after pixilation(3) (d) extracted watermark

\section{Sharpening (100) and Contrast (50)}

Simultaneously sharpening and contrast adjustment attacks are also performed. For sharpening attack, the sharpness of the watermarked host image is increased by a factor of 100 . The information is almost lost but we are still able to extract the watermark. The attacked watermarked image and the extracted watermark are shown in figure-7 (a) and (b) respectively. For Contrast Adjustment, the contrast of the watermarked host image is increased by $50 \%$. The attacked watermarked image and the extracted watermark are shown in figure-7 (c) and (d) respectively.

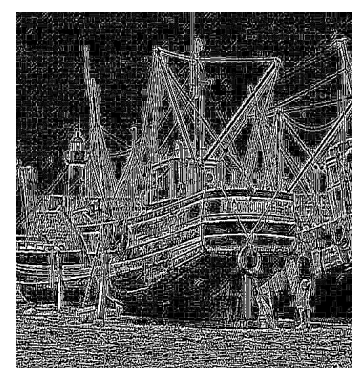

(a)

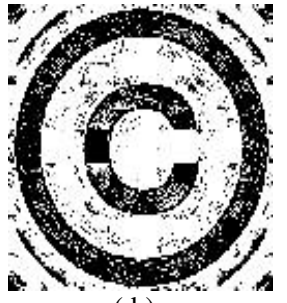

(b)

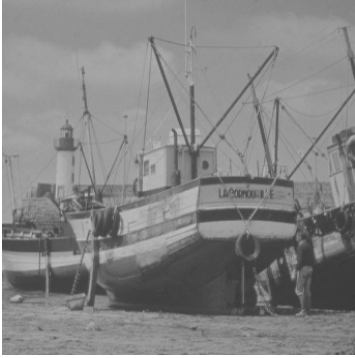

(c)

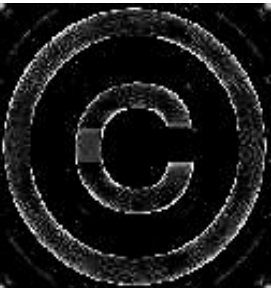

(d)

Fig 7 (a) watermarked boat image after sharpen(100)

(b) extracted watermark

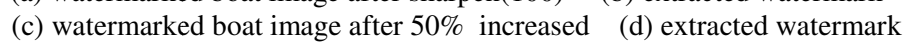

\begin{tabular}{|c|c|c|c|c|}
\hline \multirow{3}{*}{ Attacks } & \multicolumn{4}{|c|}{ Normalized cross correlation values $(\rho)$} \\
\hline & \multicolumn{3}{|c|}{ Existing methods } & \multirow{2}{*}{$\begin{array}{c}\text { Proposed Method } \\
\text { DWT-DCT-SVD }\end{array}$} \\
\hline & FrFt - SVD & DCT-SVD & DWT-SVD & \\
\hline Average Filtering $(13 \times 3)$ & -0.3163 & -0.1603 & 0.1198 & -0.0928 \\
\hline Median Filtering $(13 \times 13)$ & -0.3158 & 0.0184 & -0.0852 & -0.0852 \\
\hline $\begin{array}{c}\text { Additive Gaussian Noise } \\
(75 \%)\end{array}$ & 0.1833 & 0.2514 & 0.6749 & 0.6749 \\
\hline JPEG compression $(80: 1)$ & 0.8929 & 0.9119 & 0.9751 & 0.9751 \\
\hline $\begin{array}{l}\text { Cropping (25\% area } \\
\text { remaining) }\end{array}$ & -0.1232 & 0.1735 & 0.8810 & 0.6120 \\
\hline Resizing (512 -> 128 -> 512) & 0.4897 & -0.1036 & 0.2570 & 0.2570 \\
\hline Rotation $\left(50^{0}\right)$ & -0.5470 & 0.3702 & 0.8846 & 0.8846 \\
\hline Pixilation 3 & ------ & ------- & 0.0871 & -0.4185 \\
\hline
\end{tabular}


International Journal of Computer Science \& Information Technology (IJCSIT) Vol 4, No 2, April 2012

\begin{tabular}{|c|c|c|c|c|}
\hline Wrapping & 0.2385 & $\ldots \ldots \ldots$ & $\mathbf{0 . 7 2 9 9}$ & -0.4559 \\
\hline Histogram equalization & 0.6009 & 0.6932 & 0.9182 & 0.9182 \\
\hline Motion blur & ------- & -------- & -0.1854 & $\mathbf{- 0 . 0 3 6 3}$ \\
\hline Sharpening ( 100) & 0.1607 & 0.2758 & 0.7240 & $\mathbf{0 . 7 5 0 0}$ \\
\hline
\end{tabular}

\section{CONCLUSIONS}

In this paper we proposed a self-reference image watermarking by using the technique DWT-DCT-SVD. The watermark is visually meaningful gray scale image instead of a noise type Gaussian sequence. The proposed method is highly robust and can survive the watermark in any of attacks. The quality of the watermarked image is good in terms of perceptibility. The PSNR value for a boat image was $43.88 \mathrm{db}$. Our proposed method also provided same PSNR value. Most of the attacks provided same NCC values as in existing method [34]. But this method had well robust against motion blur and sharpening attacks, when compared with the existing method [34]. The existing method was superior to our method in averaging, pixilation and wrapping attacks. In our observations, no one can extract watermark without knowing the value of embedding depth. The future work will be carried out by embedding the watermarks in remaining other three bands.

\section{REFERENCES}

01) F. Hartung and M. Kutter, "Multimedia Watermarking Techniques," in Proceedings of the IEEE, vol. 87, no.7, pp. 1079-1107, July 1999.

02) Chi-Kwong Chan , L.M. Cheng "Hiding data in images by simple LSB substitution "Pattern Recognition 37 (2004) 469-474, ww.elsevier.com/locate/patcog.

03) Dr.M.A.Dorairangaswamy “A Robust Blind Image Watermarking Scheme in Spatial Domain for Copyright Protection” International Journal of Engineering and Technology Vol. 1, No.3, August, 2009 , ISSN: 1793-8236.

04) I. Pitas,"A method for signature casting on digital images,"Proceedings of IEEE International Conference on Image Processing," Vol. 3, pp.215-318,1996.

05) R. Wolfgang and E. Delp, ” A watermark for digital images," Proceeding of IEEE International Conference on Image Processing, Vol. 2, pp.319-222, 1996.

06) Sanghyun Joo, Youngho Suh, Jaeho Shin, and Hisakazu Kikuchi “A New Robust Watermark Embedding into Wavelet DC Components " ETRI Journal, Volume 24, Number 5, October 2002.

07) Sadi Vural, Hiromi Tomii, Hironori Yamauchi "DWT Based Robust Watermarking Embed Using CRC-32 Techniques” World Academy of Science, Engineering and Technology 52005.

08) Kundur D., and Hatzinakos, D., 'A Robust Digital Image Water- marking Scheme using Wavelet-Based fusion,' Proc. IEEE Int. Conf. On Image Processing, Santa Barbara, California, vol. 1, pp. 544-547, October 1997.

09) V. Padmanabha Reddy and Dr. S. Varadarajan “ An Effective Wavelet-Based Watermarking Scheme Using Human Visual System for Protecting Copyrights of Digital Images "International Journal of Computer and Electrical Engineering, Vol. 2, No. 1, February, 2010 ,pp1793-8163.

10) X. Xia, C.G. Boncelet, and G.R. Arce, "A Multiresolution Watermark for Digital Images,” Proc. Of IEEE ICIP, Santa Barbara, CA, USA, Oct. 1997, pp. 548-551.

11) J.R. Kim and Y.S. Moon, "A Robust Wavelet-Based Digital Watermarking Using evel-Adaptive Thresholding," Proc. Of IEEE ICIP, vol. 2, Kobe, Japan, Oct. 1999, pp. 226230. 
International Journal of Computer Science \& Information Technology (IJCSIT) Vol 4, No 2, April 2012

12) S. D. Lin and C.-F. Chen,"A Robust DCT-Based Watermarking for Copyright Protection," IEEE Transactions on Consumer Electronics, 46(3), August 2000, pp. 415-421.

13) Gengming Zhu, and Nong Sang "Watermarking Algorithm Research and Implementation Based on DCT Block " proceedings of world academy of science, engineering and technology volume 35 november 2008 issn 2070-3740

14) H. Andrews and C. Patterson, "Singular Value decompositions and Digital Image Processing," IEEE Transactions on Acoustics, Speech and Signal Processing, vol. 24, no. 1, pp. 26-53, Feb. 1976.

15) Ke-feng He, "Watermarking for images using the HVSand SVD in the wavelet domain" Proceedings of IEEEInternational Conference on Mechatronics andAutomation, pp. 2352- 2356, 2006.

16) Liu Quan, and AI Qingsong "A combination of DCT based and SVD based watermarking," ICSPproceedings of IEEE International conference on signalprocessing, pp. 873-876, 2004.

17) Alexander Sverdlov, Scott Dexter, Ahmet M. Eskicioglu "Robust SVD DCT based watermarking for copyright protection“, IEEE Transactions on Image Processing, 10(5), May 2001, pp. 724-735.

18) Feng Liu, and Yangguang Liu, "A Watermarking Algorithm for Digital Image Based on DCT and SVD,"Congress on Image and Signal Processing, pp. 380-383, 2008.

19) Liu Liang and Sun Qi, "A new SVD-DWT composite watermarking," ICSP proceedings of IEEE International conference on signal processing, 2006.

20) Gaurav Bhatnagar and Balasubramanian Raman, "Anew robust reference watermarking scheme based onDWT-SVD,” Computer Standards and Interfaces, vol.31, no. 5, pp. 1002-1013, 2009.

21) E.Ganic and A.M. Eskiciogulu et.al., Robust embedding of Visual Watermarks using DWT-SVD Journal of Electronic Imaging, October-December, 2005.

22) Q. Li, C. Yuan, and Y. Zhong, “Adaptive DWT-SVD Domain Image Watermarking Using Human Visual Model," proceedings of 9th international conference on advanced communication Technology, vol. 3, pp. 1947- 1951, Feb.2007.

23) Ali Al-Haj, and T. Manasrah, "Non-Invertible Copyright Protection of Digital Images Using DWTand SVD," Proceedings of International Conference on Digital Information Management, vol. 1, pp. $448-453,2007$.

24) S. Rezazadeh, and M. Rahmati, "A robust watermarking scheme based on wavelet transform and block SVD," 9th International Symposium on Signal Processing and Its Applications, pp. 14, 2007.

25) Swanirbhar Majumder, Madhusudhan Mishra, A. Dinamani Singh" A Hybrid SVD and Wavelet based Watermarking "2nd National Conference Mathematical Techniques: Emerging Paradigms for Electronics and IT Industries, September 26-28, 2008, pp. 197-205.

26) Humberto Ochoa , K.R. Rao “A Hybrid DWT-SVD Image-Coding System (HDWTSVD) for Color Images "systemics, cybernetics and informatics volume 1 - number 2, pp.64-70.

27) T.Sridevi, Y.Ramadevi,V.Vijaya Kumar "SECURE WATERMARKING BASED ON SVD AND WAVELETS “ICGST-GVIP Journal, Volume 10, Issue 5, December 2010.

28) S S Bedi, Ashwani Kumar, and Piyush Kapoor "Robust Secure SVD Based DCT - DWT Oriented Watermarking Technique for Image Authentication "International Conference on IT to Celebrate S. Charmonman's 72nd Birthday, March 2009, Thailand,pp 46.1-46.7.

29) K A Navas, Mathews Cheriyan Ajay, M Lekshmi, Tampy S Archana, M Sasikumar in2008 3rd International Conference on Communication Systems Software and Middleware and Workshops COMSWARE 08 (2008). 
International Journal of Computer Science \& Information Technology (IJCSIT) Vol 4, No 2, April 2012

30) V.Santhi, N. Rekha, S.Tharini "A Hybrid Block Based Watermarking Algorithm using DWTDCT-SVD Techniques for Color Images" proceedings of International Conference on Computing, Communication and Networking, 2008. ICCCn 2008.

31) Ben Wang, Jinkou Ding, Qiaoyan Wen, Xin Liao, Cuixiang Liu “An Image Watermarking Algorithm Based On DWT DCT And SVD "Proceedings of IC-NIDC2009, 978-1-4244-49002/09/\$25.00 @2009 IEEE.pp.1034-1038.

32) Yuan Xiu-Gui, Zhou Zhen “ A Novel Robust Watermarking Algorithm based on DWT-DCTSVD” computer engineering and sciences, vol 33, No 12011.

33) Satyanarayana Murty .P, P. Rajesh Kumar, " A Robust Digital Image Watermarking Scheme Using Hybrid DWT-DCT-SVD Technique,", IJCSNS pp.185-192,International Journal of Computer Science and Network Security, VOL.10 No.10, October 2010.

34) Satyanarayana Murty . P, M.Uday Bhaskar, P.Nanna Babu, P. Rajesh Kumar "A Semi-Blind Reference Watermarking Scheme Using DWT-SVD for Copyright Protection "The International Journal of Multimedia \& Its Applications (IJMA) Vol.3, No.3,pp.61-70.

\section{Biographies}

P. Satyanarayana Murty is currently working as Sr.Associate Professor in ECE Department, GIITS, Engineering College, Vishakapatnam, and Andhra Pradesh, India. $\mathrm{He}$ is working towards his Ph.D.at AU College of Engineering, Vishakapatnam, India. a. He has eighteen years experience of teaching undergraduate students and post graduate students. His research interests include image watermarking, and image compression.

Dr. P. Rajesh Kumar is currently Associate Professor in ECE Department, AU College of Engineering, Vishakapatnam, India. He received his M.Tech and Ph.D. from AndhraUniversity, Vishakapatnam, India. He has fifteen years experience of teaching. He has published 30 research papers in National and International journals. Presently he is guiding $10 \mathrm{Ph} . \mathrm{D}$ students in the area of digital signal processing and Image processing. His research interests include digital image processing and digital signal processing.
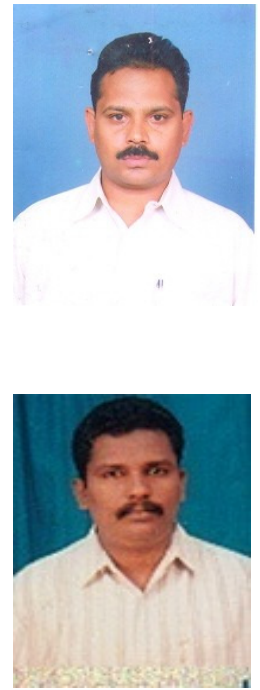